\title{
Ribose Moieties Acylation and Characterization of Some Cytidine Analogs
}

\author{
Kazi M. Rana ${ }^{\text {a }}$ Jannatul Ferdous ${ }^{\text {a }}$, \\ Anowar Hosen ${ }^{\mathrm{b}}$ and Sarkar M.A. Kawsar*a \\ ${ }^{a}$ Laboratory of Carbohydrate \\ and Nucleoside Chemistry (LCNC) \\ Department of Chemistry, Faculty of Science \\ University of Chittagong \\ Chittagong, Bangladesh \\ ${ }^{b}$ Centre for Advanced Research in Sciences \\ University of Dhaka \\ Dhaka, Bangladesh
}

Received 03.10.2020, received in revised form 10.11.2020, accepted 03.12.2020

\begin{abstract}
Modification of naturally occurring nucleosides is an important area in the search for new agents with therapeutic potential. In this study, nucleoside molecules, that is, cytidine analogs bearing ribose moieties were successfully synthesized to obtain $5^{\prime}$-O-acyl cytidine (2), which in turn was converted into 2',3'-di-O-acyl cytidine (3-7) through direct acylation. Similarly, several cytidine analogs (8-15) were formed using the aforementioned technique. Physicochemical properties and spectroscopic methods were used to characterize the newly synthesized cytidine analogs. X-ray powder diffraction was employed for quantitatively identifying crystalline compounds. Hence, these synthesized derivatives can be used as potential antimicrobial agents and promising drug candidates.
\end{abstract}

Keywords: ribose, acylation, analogs, cytidine, spectroscopy.

Citation: Rana K.M., Ferdous J., Hosen A., Kawsar S.M.A. Ribose moieties acylation and characterization of some cytidine analogs, J. Sib. Fed. Univ. Chem., 2020, 13(4), 465-478. DOI: 10.17516/1998-2836-0199

(C) Siberian Federal University. All rights reserved

This work is licensed under a Creative Commons Attribution-NonCommercial 4.0 International License (CC BY-NC 4.0).

* Corresponding author E-mail address: akawsar@cu.ac.bd 


\title{
Ацилирование рибозных фрагментов \\ и характеристики некоторых аналогов цитидина
}

\author{
К.М. Рана ${ }^{a}$, Дж. Фердоус ${ }^{a}$, \\ А. Хосен ${ }^{\boldsymbol{\sigma}}$, С.М.А. Кавсар ${ }^{\mathrm{a}}$ \\ а Лаборатория химии карбогидратов и нуклеозидов (LCNC) \\ Департамент химии, Факультет наук \\ Университет Читтагонг \\ Бангладеш, Читтагонг

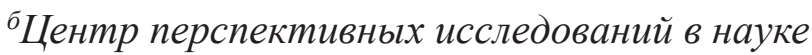 \\ Университет Дакка \\ Бангладеш, Дакка
}

Аннотация. Модификация природных нуклеозидов - важная область в поиске новых агентов с терапевтическим потенциалом. В этом исследовании нуклеозидные молекулы, являющиеся аналогами цитидина, с рибозными фрагментами были успешно использованы для синтеза 5-0-ацил цитидина (2), который в свою очередь был превращен в 2,3-ди-0-ацил цитидин (3-7) путем прямого ацилирования. Точно так же, с использованием вышеописанного метода, получено несколько аналогов цитидина (8-15). Синтезированные новые аналоги цитидина были охарактеризованы физико-химическими методами. Для количественной идентификации кристаллических соединений применили метод порошковой рентгеновской дифракции. Эти синтезированные производные могут быть использованы как потенциальные антимикробные агенты и перспективные лекарственные препараты.

Ключевые слова: рибоза, ацилирование, аналоги, цитидин, спектроскопия.

Цитирование: Рана, К.М. Ацилирование рибозных фрагментов и характеристики некоторых аналогов цитидина / К.М. Рана, Дж. Фердоус, А. Хосен, С.М.А. Кавсар // Журн. Сиб. федер. ун-та. Химия, 2020. 13(4). С. $465-478$. DOI: $10.17516 / 1998-2836-0199$

\section{Introduction}

Nucleosides and their analogues are of enormous importance. They are an established class of clinically useful medicinal agents, possessing antiviral and anticancer activity [1-3]. A number of different types of nucleosides have been synthesized from time to time which are reported to be biologically active, for example, ribavirin or virazole is an important such nucleoside. It has been reported as one of the most powerful synthetic antiviral agent's active against DNA viruses [4]. Virazole has been approved by the FDA for the treatment of viral infections [5]. The 5-azacytidine has been shown to possess promising activity against adult non-lymphocytic leukemia. It can either be synthesized chemically or produced microbiologically. It mainly affects the synthesis and function of DNA [6]. 2',3'-Dideoxycytidine also been reported to inhibit HIV and its clinical trials have been successfully carried out at NIH in AIDS patients [7]. Structural modifications of nucleosides have 


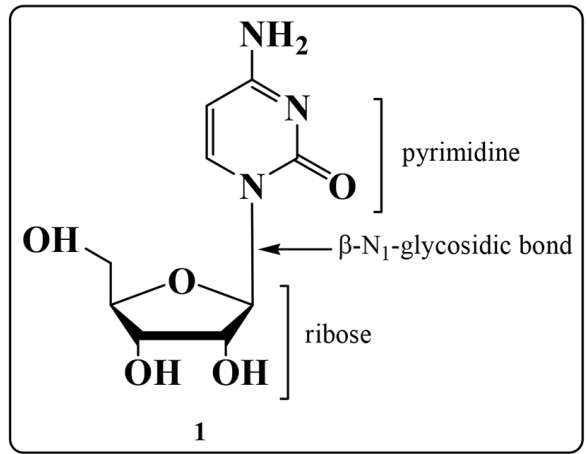

Fig. 1. Structure of cytidine

given rise to widely used drugs such as zidovudine [8] and acyclovir [9], which demonstrates that this strategy offers interesting opportunities to synthesize new therapeutically useful compounds.

Cytidine (Fig. 1) is a nucleoside that consists of a sugar part, ribose, which is linked to the pyrimidine base cytosine via a $\beta$-glycosidic bond. Cytidine is a component of RNA and a precursor for uridine. When RNA-rich food is consumed, RNA is broken down into ribosyl pyrimidines (cytidine and uridine) and its basic elements are released for absorption from intestine [10]. RNA-rich foods are considered good cytidine sources. The supplementation of dietary cytidine (5')-diphosphocholine protects against the development of memory deficits [11].

Modifications in the sugar moiety of nucleosides have resulted in various effective therapeutic applications. In the last few years, many researchers have investigated the selective acylation of the hydroxyl groups of the ribose moieties of nucleosides and nucleotides by using various methods $[12,13]$. Different methods for nucleoside acylation have been successfully developed and employed $[14,15]$. Among those, a direct method is most encouraging for nucleoside acylation [16].

Encouraged by the literature [17, 18] and our findings [19-21], we synthesized some selectively acylated analogs of cytidine (schemes 1-4) containing various substituents in a single molecular framework and X-ray diffraction studies on them for the first time.

\section{Experimental}

\section{Materials and methods}

Thin layer chromatography (TLC) was performed on Kieselgel $\mathrm{GF}_{254}$, and visualization was achieved by spraying plates with $1 \% \mathrm{H}_{2} \mathrm{SO}_{4}$ followed by heating the plates at $150-200{ }^{\circ} \mathrm{C}$ until coloration occurred. Melting points (m.p.) were determined using an electrothermal melting point apparatus and were uncorrected. Evaporation was performed using a Büchi rotary evaporator under diminished pressure. Analytical grade solvents were employed and purified using standard procedures. Infrared (IR) spectral analyses were conducted using a Fourier transform IR (FTIR) spectrophotometer (IR Prestige-21, Shimadzu, Japan) within $200-4000 \mathrm{~cm}^{-1}$ at the Department of Chemistry, University of Chittagong, Bangladesh. The mass spectra of the synthesized compounds were recorded through liquid chromatography electrospray ionization-tandem mass spectrometry in the positive ionization mode (LC/ESI(+)-MS/MS) by using a system comprising a JASSO LC (JASCO, Japan). A Brucker advance DPX $400 \mathrm{MHz}$ with tetramethylsilane as an internal standard was used to record ${ }^{1} \mathrm{H}-\mathrm{NMR}$

$$
-467-
$$


spectra in $\mathrm{CDCl}_{3}$ ( $\delta$ in ppm) at WMSRC, JU, Bangladesh. XRD patterns were obtained using an XRD-53 analyzer, Rigaku, Japan diffractometer, with a back monochromator and $\mathrm{Cu}$ target and $\mathrm{K} \alpha$ $(\lambda=1.5406 \mathrm{~nm})$ in $2 \theta=2^{\circ}-70^{\circ}$ at CARS, Dhaka University, Bangladesh. Column chromatography was performed with silica gel $\mathrm{G}_{60} \cdot \mathrm{CHCl}_{3} / \mathrm{CH}_{3} \mathrm{OH}$ was employed as the solvent system for TLC analyses was in different proportions. All reagents used were commercially available (Aldrich) and used without further purification unless otherwise specified.

\section{Synthesis}

Over past several years, our laboratory has been synthesizing nucleoside derivatives containing various acyl groups to explore their antimicrobial properties [30, 31].

In dry DMF ( $N, N$-dimethylformamide) ( $3 \mathrm{ml})$, a cytidine (1) $(70 \mathrm{mg}, 0.287 \mathrm{mmol})$ solution was cooled to $-5{ }^{\circ} \mathrm{C}$ when decanoyl chloride (65 mg, 1.1 molar eq.) was added. The solution was stirred at this temperature for 5 to $6 \mathrm{~h}$ and then was allowed to stand at room temperature overnight. Reaction progress was monitored through TLC, which indicated the complete conversion of the starting material into a single product. A few pieces of ice were added to the flask to stop the reaction. Subsequently, the solvent was evaporated using a high pressure vacuum evaporator, and the resulted product was passed through silica gel column chromatography and eluted with (1:24), which provided the decanoyl derivative (2) (92 mg). The recrystallization of $\left(\mathrm{CHCl}_{3}-\mathrm{C}_{6} \mathrm{H}_{14}\right)$ led to the formation of the title derivative (2) as needles. The compound was sufficiently pure for subsequent use without further treatment and identification.

5'-O-Decanoylcytidine (2): Yield $83.8 \%$ as crystalline solid, M.P. $85-87{ }^{\circ} \mathrm{C}\left(\mathrm{CHCl}_{3}-\mathrm{C}_{6} \mathrm{H}_{14}\right)$, $\mathrm{R}_{f}=0.51\left(\mathrm{CHCl}_{3} / \mathrm{CH}_{3} \mathrm{OH}=24 / 1\right.$, v/v). FTIR: $v_{\max }$ 1731, $1714(-\mathrm{CO}), 3550(-\mathrm{NH}), 3420 \mathrm{~cm}^{-1}(-\mathrm{OH})$. ${ }^{1} \mathrm{H}-\mathrm{NMR}\left(400 \mathrm{MHz}, \mathrm{CDCl}_{3}\right): \delta_{\mathrm{H}} 9.02(1 \mathrm{H}, \mathrm{s},-\mathrm{NH}), 7.44(1 \mathrm{H}, \mathrm{d}, \mathrm{J}=7.8 \mathrm{~Hz}, \mathrm{H}-6), 6.56(1 \mathrm{H}, \mathrm{d}, \mathrm{J}=3.0 \mathrm{~Hz}$, H-1'), $6.49\left(1 \mathrm{H}, \mathrm{s}, 2^{\prime}-\mathrm{OH}\right), 6.01\left(1 \mathrm{H}, \mathrm{dd}, \mathrm{J}=2.4\right.$ and $\left.12.3 \mathrm{~Hz}, \mathrm{H}-5^{\prime} \mathrm{a}\right), 5.56(1 \mathrm{H}, \mathrm{dd}, \mathrm{J}=2.4$ and $12.3 \mathrm{~Hz}$, H-5'b), $5.45\left(1 \mathrm{H}, \mathrm{s}, 3^{\prime}-\mathrm{OH}\right), 4.85\left(1 \mathrm{H}, \mathrm{dd}, \mathrm{J}=2.4\right.$ and $\left.5.6 \mathrm{~Hz}, \mathrm{H}-4^{\prime}\right), 4.60\left(2 \mathrm{H}, \mathrm{s},-\mathrm{NH}_{2}\right), 4.45(1 \mathrm{H}, \mathrm{d}$, $\left.\mathrm{J}=3.2 \mathrm{~Hz}, \mathrm{H}-2^{\prime}\right), 4.31\left(1 \mathrm{H}, \mathrm{dd}, \mathrm{J}=3.6\right.$ and $\left.5.8 \mathrm{~Hz}, \mathrm{H}-3^{\prime}\right), 3.80(1 \mathrm{H}, \mathrm{d}, \mathrm{J}=7.2 \mathrm{~Hz}, \mathrm{H}-5), 2.38\{2 \mathrm{H}$, $\left.\mathrm{m}, \mathrm{CH}_{3}\left(\mathrm{CH}_{2}\right)_{7} \mathrm{CH}_{2} \mathrm{CO}-\right\}, 1.59\left\{2 \mathrm{H}, \mathrm{m}, \mathrm{CH}_{3}\left(\mathrm{CH}_{2}\right)_{6} \mathrm{CH}_{2} \mathrm{CH}_{2} \mathrm{CO}-\right\}, 1.32\left\{12 \mathrm{H}, \mathrm{m}, \mathrm{CH}_{3}\left(\mathrm{CH}_{2}\right)_{6}\left(\mathrm{CH}_{2}\right)_{2} \mathrm{CO}-\right\}$, $0.85\left\{3 \mathrm{H}, \mathrm{m}, \mathrm{CH}_{3}\left(\mathrm{CH}_{2}\right)_{8} \mathrm{CO}-\right\}$. MS [M+1] 398.10 .

Anal Calcd. for $\mathrm{C}_{19} \mathrm{H}_{31} \mathrm{~N}_{3} \mathrm{O}_{6}$ : \% C, 72.54, H, 7.81; found: \% C, 72.53, H, 7.82.

\section{General procedure for the direct 2', $\mathbf{3}^{\prime}$-di-O-acylation of 5 '-O-decanoylcytidine (2) derivatives (3-7)}

In DMF $(3 \mathrm{ml})$, octanoyl chloride $(0.185 \mathrm{ml}, 4$ molar eq. $)$ was added to a cooled $\left(0{ }^{\circ} \mathrm{C}\right)$ and stirred solution of the decanoyl derivative (2) $(110 \mathrm{mg}, 0.276 \mathrm{mmol})$. The mixture was stirred at $0{ }^{\circ} \mathrm{C}$ for $8 \mathrm{~h}$ and then allowed to stand overnight at room temperature. TLC analyses showed the complete conversion of reactants into a single product. A few pieces of ice were added to the reaction flask to eliminate the excess reagent, and the reaction mixture was evaporated using the high-pressure vacuum evaporator to remove the solvent. The percolation of the resulting product achieved by passing through a silica gel column with the $\mathrm{CHCl}_{3}-\mathrm{CH}_{3} \mathrm{OH}$ eluant led to the formation of the octanoyl derivative (3) (163 $\mathrm{mg}$ ) as a crystalline solid.

A similar reaction and purification procedure was employed to prepare compounds (4), (5), (6), and (7). 
5'-O-Decanoyl-2',3'-di-O-octanoylcytidine (3): Yield $77.6 \%$ as a white crystalline solid, M.P. $89-91{ }^{\circ} \mathrm{C}\left(\mathrm{CHCl}_{3}-\mathrm{C}_{6} \mathrm{H}_{14}\right), \mathrm{R}_{f}=0.51\left(\mathrm{CHCl}_{3} / \mathrm{CH}_{3} \mathrm{OH}=24 / 1\right.$, v/v). FTIR: $v_{\max } 1729,1716(-\mathrm{CO}), 3470 \mathrm{~cm}^{-1}$ (-NH). ${ }^{1} \mathrm{H}-\mathrm{NMR}\left(400 \mathrm{MHz}, \mathrm{CDCl}_{3}\right): \delta_{\mathrm{H}} 9.18(1 \mathrm{H}, \mathrm{s},-\mathrm{NH}), 7.27(1 \mathrm{H}, \mathrm{d}, \mathrm{J}=7.8 \mathrm{~Hz}, \mathrm{H}-6), 6.54(1 \mathrm{H}, \mathrm{d}$, $\left.\mathrm{J}=3.2 \mathrm{~Hz}, \mathrm{H}-1^{\prime}\right), 5.45\left(1 \mathrm{H}, \mathrm{m}, \mathrm{H}-2^{\prime}\right), 4.82\left(1 \mathrm{H}, \mathrm{dd}, \mathrm{J}=3.5\right.$ and $\left.5.6 \mathrm{~Hz}, \mathrm{H}-3^{\prime}\right), 4.67(1 \mathrm{H}, \mathrm{dd}, \mathrm{J}=2.4$ and $\left.12.2 \mathrm{~Hz}, \mathrm{H}-5^{\prime} \mathrm{a}\right), 4.58\left(2 \mathrm{H}, \mathrm{s},-\mathrm{NH}_{2}\right), 4.55\left(1 \mathrm{H}, \mathrm{dd}, \mathrm{J}=2.4\right.$ and $\left.12.2 \mathrm{~Hz}, \mathrm{H}-5^{\prime} \mathrm{b}\right), 4.38(1 \mathrm{H}, \mathrm{dd}, \mathrm{J}=2.4$ and $\left.5.5 \mathrm{~Hz}, \mathrm{H}-4^{\prime}\right), 3.70(1 \mathrm{H}, \mathrm{d}, \mathrm{J}=7.2 \mathrm{~Hz}, \mathrm{H}-5), 2.37\left\{4 \mathrm{H}, \mathrm{m}, 2 \times \mathrm{CH}_{3}\left(\mathrm{CH}_{2}\right)_{5} \mathrm{CH} \mathrm{H}_{2} \mathrm{CO}-\right\}, 1.64\{4 \mathrm{H}, \mathrm{m}$, $\left.2 \times \mathrm{CH}_{3}\left(\mathrm{CH}_{2}\right)_{4} \mathrm{CH}_{2} \mathrm{CH}_{2} \mathrm{CO}-\right\}, 1.29\left\{16 \mathrm{H}, \mathrm{m}, 2 \times \mathrm{CH}_{3}\left(\mathrm{CH}_{2}\right)_{4}\left(\mathrm{CH}_{2}\right)_{2} \mathrm{CO}-\right\}, 0.89\left\{6 \mathrm{H}, \mathrm{m}, 2 \times \mathrm{CH}_{3}\left(\mathrm{CH}_{2}\right)_{6} \mathrm{CO}-\right\}$. MS $[\mathrm{M}+1]^{+} 650.09$.

Anal Calcd. for $\mathrm{C}_{35} \mathrm{H}_{59} \mathrm{O}_{8} \mathrm{~N}_{3}$ : \% C, 64.71, H, 9.09; \% found: C, 64.73, H, 9.06.

5'-O-Decanoyl-2',3'-di- $\boldsymbol{O}$-palmitoylcytidine (4): Yield $91.2 \%$ as a white crystalline solid, M.P. $78-79{ }^{\circ} \mathrm{C}\left(\mathrm{CHCl}_{3}-\mathrm{C}_{6} \mathrm{H}_{14}\right), \mathrm{R}_{f}=0.54\left(\mathrm{CHCl}_{3} / \mathrm{CH}_{3} \mathrm{OH}=24 / 1\right.$, v/v). FTIR: $v_{\max } 1696(-\mathrm{CO}), 3500 \mathrm{~cm}^{-1}$ $(-\mathrm{NH}) .{ }^{1} \mathrm{H}-\mathrm{NMR}\left(400 \mathrm{MHz}, \mathrm{CDCl}_{3}\right): \delta_{\mathrm{H}} 9.10(1 \mathrm{H}, \mathrm{s},-\mathrm{NH}), 7.29(1 \mathrm{H}, \mathrm{d}, \mathrm{J}=7.7 \mathrm{~Hz}, \mathrm{H}-6), 6.52(1 \mathrm{H}$, d, J = 3.2 Hz, H-1'), $5.48\left(1 \mathrm{H}, \mathrm{m}, \mathrm{H}-2^{\prime}\right), 4.86\left(1 \mathrm{H}, \mathrm{dd}, \mathrm{J}=3.5\right.$ and $\left.5.6 \mathrm{~Hz}, \mathrm{H}-3^{\prime}\right), 4.67(1 \mathrm{H}, \mathrm{dd}, \mathrm{J}=2.4$ and $\left.12.1 \mathrm{~Hz}, \mathrm{H}-5^{\prime} \mathrm{a}\right), 4.58\left(2 \mathrm{H}, \mathrm{s},-\mathrm{NH}_{2}\right), 4.56\left(1 \mathrm{H}, \mathrm{dd}, \mathrm{J}=2.4\right.$ and $\left.12.1 \mathrm{~Hz}, \mathrm{H}-5^{\prime} \mathrm{b}\right), 4.41(1 \mathrm{H}$, dd, $\mathrm{J}=2.4$ and $\left.5.5 \mathrm{~Hz}, \mathrm{H}-4^{\prime}\right), 3.81(1 \mathrm{H}, \mathrm{m}, \mathrm{H}-5), 2.36\left\{4 \mathrm{H}, \mathrm{m}, 2 \times \mathrm{CH}_{3}\left(\mathrm{CH}_{2}\right)_{13} \mathrm{CH} \mathrm{H}_{2} \mathrm{CO}-\right\}, 1.27\{52 \mathrm{H}, \mathrm{m}$, $\left.2 \times \mathrm{CH}_{3}\left(\mathrm{CH}_{2}\right)_{13} \mathrm{CH}_{2} \mathrm{CO}-\right\}, 0.91\left\{6 \mathrm{H}, \mathrm{m}, 2 \times \mathrm{CH}_{3}\left(\mathrm{CH}_{2}\right)_{14} \mathrm{CO}-\right\}$. MS [M+1] $]^{+} 874.30$.

Anal Calcd. for $\mathrm{C}_{51} \mathrm{H}_{91} \mathrm{O}_{8} \mathrm{~N}_{3}$ : \% C, 70.10, H, 10.42; found: \% C, 70.13, H, 10.40 .

$\mathbf{5}^{\prime}$ - $\boldsymbol{O}$-Decanoyl-2',3'-di- $\boldsymbol{O}$-stearoylcytidine (5): Yield $85.3 \%$ as crystalline solid, M.P. $95-$ $97{ }^{\circ} \mathrm{C}\left(\mathrm{CHCl}_{3}-\mathrm{C}_{6} \mathrm{H}_{14}\right), \mathrm{R}_{f}=0.50\left(\mathrm{CHCl}_{3} / \mathrm{CH}_{3} \mathrm{OH}=23 / 1, \mathrm{v} / \mathrm{v}\right)$. FTIR: $v_{\max } 1709(-\mathrm{CO}), 3490 \mathrm{~cm}^{-1}$ (-NH). ${ }^{1} \mathrm{H}-\mathrm{NMR}\left(400 \mathrm{MHz}, \mathrm{CDCl}_{3}\right): \delta_{\mathrm{H}} 9.08(1 \mathrm{H}, \mathrm{s},-\mathrm{NH}), 7.28(1 \mathrm{H}, \mathrm{d}, \mathrm{J}=7.7 \mathrm{~Hz}, \mathrm{H}-6), 6.68(1 \mathrm{H}$, $\left.\mathrm{d}, \mathrm{J}=3.2 \mathrm{~Hz}, \mathrm{H}-1^{\prime}\right), 6.48\left(1 \mathrm{H}, \mathrm{m}, \mathrm{H}-2^{\prime}\right), 6.21\left(1 \mathrm{H}, \mathrm{dd}, \mathrm{J}=3.5\right.$ and $\left.5.6 \mathrm{~Hz}, \mathrm{H}-3^{\prime}\right), 6.07(1 \mathrm{H}, \mathrm{dd}$, $\mathrm{J}=2.4$ and $\left.12.1 \mathrm{~Hz}, \mathrm{H}-5^{\prime} \mathrm{a}\right), 5.51\left(1 \mathrm{H}, \mathrm{dd}, \mathrm{J}=2.4\right.$ and $\left.12.1 \mathrm{~Hz}, \mathrm{H}-5^{\prime} \mathrm{b}\right), 5.21(1 \mathrm{H}, \mathrm{dd}, \mathrm{J}=2.4$ and $\left.5.5 \mathrm{~Hz}, \mathrm{H}-4^{\prime}\right), 4.88\left(2 \mathrm{H}, \mathrm{s},-\mathrm{NH}_{2}\right), 4.11(1 \mathrm{H}, \mathrm{m}, \mathrm{H}-5), 2.36\left\{4 \mathrm{H}, \mathrm{m}, 2 \times \mathrm{CH}_{3}\left(\mathrm{CH}_{2}\right)_{15} \mathrm{CH}_{2} \mathrm{CO}-\right\}, 1.65$ $\left\{4 \mathrm{H}, \mathrm{m}, 2 \times \mathrm{CH}_{3}\left(\mathrm{CH}_{2}\right)_{14} \mathrm{CH}_{2} \mathrm{CH}_{2} \mathrm{CO}\right\}, 1.27\left\{56 \mathrm{H}, \mathrm{m}, 2 \times \mathrm{CH}_{3}\left(\mathrm{CH}_{2}\right)_{14} \mathrm{CH}_{2} \mathrm{CH}_{2} \mathrm{CO}-\right\}, 0.90\{6 \mathrm{H}, \mathrm{m}$, $\left.2 \times \mathrm{CH}_{3}\left(\mathrm{CH}_{2}\right)_{16} \mathrm{CO}-\right\}$. MS [M+1] $]^{+} 930.23$.

Anal Calcd. for $\mathrm{C}_{55} \mathrm{H}_{99} \mathrm{O}_{8} \mathrm{~N}_{3}: \%$ C, 71.0, H, 10.60; \% found: C, 71.03, H, 10.63 .

5'-O-Decanoyl-2',3'-di- $\boldsymbol{O}$-(triphenylmethyl)cytidine (6): Yield $84.3 \%$ as a white crystalline solid, M.P. $102-104{ }^{\circ} \mathrm{C}\left(\mathrm{CHCl}_{3}-\mathrm{C}_{6} \mathrm{H}_{14}\right), \mathrm{R}_{f}=0.52\left(\mathrm{CHCl}_{3} / \mathrm{CH}_{3} \mathrm{OH}=22 / 1\right.$, v/v). FTIR: $v_{\max } 1686(-\mathrm{CO})$, $3496 \mathrm{~cm}^{-1}(-\mathrm{NH}) .{ }^{1} \mathrm{H}-\mathrm{NMR}\left(400 \mathrm{MHz}, \mathrm{CDCl}_{3}\right): \delta_{\mathrm{H}} 9.02(1 \mathrm{H}, \mathrm{s},-\mathrm{NH}), 7.58(12 \mathrm{H}, \mathrm{m}, 2 \times \mathrm{Ar}-\mathrm{H}), 7.36(18 \mathrm{H}$, m, 2×Ar-H), $7.22(1 \mathrm{H}, \mathrm{d}, \mathrm{J}=7.6 \mathrm{~Hz}, \mathrm{H}-6), 6.61\left(1 \mathrm{H}, \mathrm{d}, \mathrm{J}=3.2 \mathrm{~Hz}, \mathrm{H}-1^{\prime}\right), 5.47\left(1 \mathrm{H}, \mathrm{d}, \mathrm{J}=3.2 \mathrm{~Hz}, \mathrm{H}-2^{\prime}\right)$, $4.88\left(1 \mathrm{H}, \mathrm{dd}, \mathrm{J}=3.6\right.$ and $\left.5.6 \mathrm{~Hz}, \mathrm{H}-3^{\prime}\right), 4.68\left(1 \mathrm{H}, \mathrm{dd}, \mathrm{J}=2.3\right.$ and $\left.12.1 \mathrm{~Hz}, \mathrm{H}-5^{\prime} \mathrm{a}\right), 4.60\left(2 \mathrm{H}, \mathrm{s},-\mathrm{NH}_{2}\right)$, $4.57\left(1 \mathrm{H}, \mathrm{dd}, \mathrm{J}=2.4\right.$ and $\left.12.1 \mathrm{~Hz}, \mathrm{H}-5^{\prime} \mathrm{b}\right), 4.49\left(1 \mathrm{H}, \mathrm{dd}, \mathrm{J}=2.4\right.$ and $\left.5.5 \mathrm{~Hz}, \mathrm{H}-4^{\prime}\right), 3.92(1 \mathrm{H}, \mathrm{m}, \mathrm{H}-5)$. MS $[\mathrm{M}+1]^{+} 882.12$.

Anal Calcd. for $\mathrm{C}_{57} \mathrm{H}_{59} \mathrm{O}_{6} \mathrm{~N}_{3}$ : \% C, 77.60, H, 6.71; \% found: C, 77.62, H, 6.73.

5'-O-Decanoyl-2',3'-(4-tert-butylbenzoyl)cytidine (7): Yield 90.4\% as crystalline solid, M.P. $109-111{ }^{\circ} \mathrm{C}\left(\mathrm{CHCl}_{3}-\mathrm{C}_{6} \mathrm{H}_{14}\right), \mathrm{R}_{f}=0.52\left(\mathrm{CHCl}_{3} / \mathrm{CH}_{3} \mathrm{OH}=24 / 1\right.$, v/v). FTIR: v $v_{\max } 1712(-\mathrm{CO}), 3501 \mathrm{~cm}^{-1}$ (-NH). ${ }^{1} \mathrm{H}-\mathrm{NMR}\left(400 \mathrm{MHz}, \mathrm{CDCl}_{3}\right): \delta_{\mathrm{H}} 9.03(1 \mathrm{H}, \mathrm{s},-\mathrm{NH}), 8.06(4 \mathrm{H}, \mathrm{m}, 2 \times \mathrm{Ar}-\mathrm{H}), 7.51(4 \mathrm{H}, \mathrm{m}, 2 \times \mathrm{Ar}-\mathrm{H})$, $7.28(1 \mathrm{H}, \mathrm{d}, \mathrm{J}=7.7 \mathrm{~Hz}, \mathrm{H}-6), 6.68\left(1 \mathrm{H}, \mathrm{d}, \mathrm{J}=3.2 \mathrm{~Hz}, \mathrm{H}-1^{\prime}\right), 5.57\left(1 \mathrm{H}, \mathrm{m}, \mathrm{H}-2^{\prime}\right), 4.96(1 \mathrm{H}, \mathrm{dd}, \mathrm{J}=3.5$ and $\left.5.6 \mathrm{~Hz}, \mathrm{H}-3^{\prime}\right), 4.66\left(1 \mathrm{H}, \mathrm{dd}, \mathrm{J}=2.4\right.$ and $\left.12.1 \mathrm{~Hz}, \mathrm{H}-5^{\prime} \mathrm{a}\right), 4.54\left(2 \mathrm{H}, \mathrm{s},-\mathrm{NH}_{2}\right), 4.52(1 \mathrm{H}, \mathrm{dd}, \mathrm{J}=2.4$ and $\left.12.1 \mathrm{~Hz}, \mathrm{H}-5^{\prime} \mathrm{b}\right), 4.25\left(1 \mathrm{H}, \mathrm{dd}, \mathrm{J}=2.4\right.$ and $\left.5.5 \mathrm{~Hz}, \mathrm{H}-4^{\prime}\right), 4.01(1 \mathrm{H}, \mathrm{m}, \mathrm{H}-5), 1.33,1.28\{18 \mathrm{H}, 2 \times \mathrm{s}$, $\left.2 \times\left(\mathrm{CH}_{3}\right)_{3} \mathrm{C}-\right\}$. MS $[\mathrm{M}+1]^{+} 718.11$.

Anal Calcd. for $\mathrm{C}_{41} \mathrm{H}_{55} \mathrm{O}_{8} \mathrm{~N}_{3}$ : \% C, 68.60, H, 7.60; \% found: C, 68.63, H, 7.58. 
In dry DMF (3 ml), a cytidine (1) $(70 \mathrm{mg}, 0.287 \mathrm{mmol})$ solution was cooled to $-5{ }^{\circ} \mathrm{C}$ and treated with 1.1 molar equivalent of triphenylmethyl chloride $(85 \mathrm{mg})$ with continuous stirring at the same temperature for 5 to $6 \mathrm{~h}$. Stirring was continued overnight at room temperature. Reaction progress was monitored through TLC. A few pieces of ice were added to the flask to terminate the reaction. Subsequently, the solvent was evaporated using the high-pressure vacuum evaporator. The resulting syrupy mass was purified through silica gel column chromatography (with $\mathrm{CHCl}_{3}-\mathrm{CH}_{3} \mathrm{OH}, 1: 24$ eluant) to acquire the title compound $(\mathbf{8}, 107 \mathrm{mg})$ as a crystalline solid.

$\mathbf{5}^{\prime}$-O -(Triphenylmethyl)cytidine (8): Yield $81.2 \%$ as a white crystalline solid, M.P. $75-77^{\circ} \mathrm{C}$ ) $\left(\mathrm{CHCl}_{3}-\mathrm{C}_{6} \mathrm{H}_{14}\right), \mathrm{R}_{f}=0.50\left(\mathrm{CHCl}_{3} / \mathrm{CH}_{3} \mathrm{OH}=24 / 1, \mathrm{v} / \mathrm{v}\right)$. FTIR: $v_{\max } 1701(-\mathrm{CO}), 3547(-\mathrm{NH}), 3416-3468$ (br) $\mathrm{cm}^{-1}(-\mathrm{OH}) .{ }^{1} \mathrm{H}-\mathrm{NMR}\left(400 \mathrm{MHz}, \mathrm{CDCl}_{3}\right): \delta_{\mathrm{H}} 9.01(1 \mathrm{H}, \mathrm{s},-\mathrm{NH}), 7.35(6 \mathrm{H}, \mathrm{m}, \mathrm{Ar}-\mathrm{H}), 7.30(9 \mathrm{H}, \mathrm{m}$, Ar-H), $7.23(1 \mathrm{H}, \mathrm{d}, \mathrm{J}=7.8 \mathrm{~Hz}, \mathrm{H}-6), 6.61\left(1 \mathrm{H}, \mathrm{d}, \mathrm{J}=3.0 \mathrm{~Hz}, \mathrm{H}-1^{\prime}\right), 6.48\left(1 \mathrm{H}, \mathrm{s}, 2^{\prime}-\mathrm{OH}\right), 5.30$ (1H, dd, $\mathrm{J}=2.2$ and $\left.12.2 \mathrm{~Hz}, \mathrm{H}-5^{\prime} \mathrm{a}\right), 5.26\left(1 \mathrm{H}, \mathrm{dd}, \mathrm{J}=2.2\right.$ and $\left.12.2 \mathrm{~Hz}, \mathrm{H}-5^{\prime} \mathrm{b}\right), 5.15\left(1 \mathrm{H}, \mathrm{s}, 3^{\prime}-\mathrm{OH}\right), 4.85(1 \mathrm{H}, \mathrm{dd}$, $\mathrm{J}=2.2$ and $\left.5.6 \mathrm{~Hz}, \mathrm{H}-4^{\prime}\right), 4.50\left(2 \mathrm{H}, \mathrm{s},-\mathrm{NH}_{2}\right), 4.25\left(1 \mathrm{H}, \mathrm{d}, \mathrm{J}=3.2 \mathrm{~Hz}, \mathrm{H}-2^{\prime}\right), 4.01(1 \mathrm{H}, \mathrm{dd}, \mathrm{J}=3.6$ and $\left.5.8 \mathrm{~Hz}, \mathrm{H}-3^{\prime}\right), 3.91(1 \mathrm{H}, \mathrm{d}, \mathrm{J}=7.2 \mathrm{~Hz}, \mathrm{H}-5)$. MS [M+1] 486.08 .

Anal Calcd. for $\mathrm{C}_{28} \mathrm{H}_{27} \mathrm{O}_{5} \mathrm{~N}_{3}$ : \% C, 69.28, H, 5.56; \% found: C, 69.27, H, 5.53.

\section{General procedure for the direct 2',3'-di-O-acylation of $5^{\prime}$-O-(triphenylmethyl)cytidine derivatives (9-15)}

The triphenylmethyl derivative $(\mathbf{8}, 60 \mathrm{mg}, 0.124 \mathrm{mmol})$ was dissolved in dry DMF (3 ml), and the solution was cooled to $0{ }^{\circ} \mathrm{C}$ when hexanoyl chloride $(0.067 \mathrm{ml}, 4$ molar eq. $)$ was added. The mixture was stirred at $0{ }^{\circ} \mathrm{C}$ for $6 \mathrm{~h}$ and at room temperature overnight. The conventional work-up procedure and subsequent chromatographic purification with the $\mathrm{CHCl}_{3}-\mathrm{CH}_{3} \mathrm{OH}(1: 24)$ eluant led to the formation of 2',3'-di- $O$-hexanoyl derivative (9) (157 mg).

$\mathbf{2}^{\prime}, \mathbf{3}^{\prime}$-Di- $\boldsymbol{O}$-hexanoyl-5' $\boldsymbol{O} \boldsymbol{O}$-(triphenylmethyl)cytidine (9): Yield $81.5 \%$ as crystalline solid, M.P. $100-102{ }^{\circ} \mathrm{C}, \mathrm{R}_{f}=0.52\left(\mathrm{CHCl}_{3} / \mathrm{CH}_{3} \mathrm{OH}=22 / 1\right.$, v/v). FTIR: $v_{\max } 1710(-\mathrm{CO}), 3503 \mathrm{~cm}^{-1}(-\mathrm{NH}) .{ }^{1} \mathrm{H}-\mathrm{NMR}$ $\left(400 \mathrm{MHz}, \mathrm{CDCl}_{3}\right): \delta_{\mathrm{H}} 9.08(1 \mathrm{H}, \mathrm{s},-\mathrm{NH}), 7.32(6 \mathrm{H}, \mathrm{m}, \mathrm{Ar}-\mathrm{H}), 7.28(9 \mathrm{H}, \mathrm{m}, \mathrm{Ar}-\mathrm{H}), 7.21(1 \mathrm{H}, \mathrm{d}, \mathrm{J}=7.6 \mathrm{~Hz}$, H-6), $6.51\left(1 \mathrm{H}, \mathrm{d}, \mathrm{J}=3.2 \mathrm{~Hz}, \mathrm{H}-1^{\prime}\right), 5.47\left(1 \mathrm{H}, \mathrm{m}, \mathrm{H}-2^{\prime}\right), 4.88\left(1 \mathrm{H}, \mathrm{dd}, \mathrm{J}=3.2\right.$ and $\left.5.4 \mathrm{~Hz}, \mathrm{H}-3^{\prime}\right), 4.61$ $\left(1 \mathrm{H}, \mathrm{dd}, \mathrm{J}=2.2\right.$ and $\left.12.2 \mathrm{~Hz}, \mathrm{H}-5^{\prime} \mathrm{a}\right), 4.54\left(2 \mathrm{H}, \mathrm{s},-\mathrm{NH}_{2}\right), 4.48\left(1 \mathrm{H}, \mathrm{dd}, \mathrm{J}=2.2\right.$ and $\left.12.2 \mathrm{~Hz}, \mathrm{H}-5^{\prime} \mathrm{b}\right), 4.35$ $\left(1 \mathrm{H}, \mathrm{dd}, \mathrm{J}=2.2\right.$ and $\left.5.4 \mathrm{~Hz}, \mathrm{H}-4^{\prime}\right), 3.81(1 \mathrm{H}, \mathrm{d}, \mathrm{J}=7.1 \mathrm{~Hz}, \mathrm{H}-5), 2.31\left\{4 \mathrm{H}, \mathrm{m}, 2 \times \mathrm{CH}_{3}\left(\mathrm{CH}_{2}\right)_{3} \mathrm{CH}_{2} \mathrm{CO}-\right\}$, $1.62\left\{4 \mathrm{H}, \mathrm{m}, 2 \times \mathrm{CH}_{3}\left(\mathrm{CH}_{2}\right)_{2} \mathrm{CH}_{2} \mathrm{CH}_{2} \mathrm{CO}-\right\}, 1.26\left\{8 \mathrm{H}, \mathrm{m}, 2 \times \mathrm{CH}_{3}\left(\mathrm{CH}_{2}\right)_{2} \mathrm{CH}_{2} \mathrm{CH}_{2} \mathrm{CO}-\right\}, 0.88\{6 \mathrm{H}, \mathrm{m}$, $\left.2 \times \mathrm{CH}_{3}\left(\mathrm{CH}_{2}\right)_{4} \mathrm{CO}-\right\}$. MS [M+1] $]^{+} 682.05$.

Anal Calcd. for $\mathrm{C}_{40} \mathrm{H}_{47} \mathrm{O}_{7} \mathrm{~N}_{3}$ : \% C, 70.40, H, 6.90; \% found: C, 70.42, H, 6.91.

A similar reaction and purification method were employed to synthesize compounds (10) (164 mg), (11) (152 mg), (12) (109.5 mg), (13) (100.4 mg), (14) (132 mg), and (15) (111 mg).

2', 3'-Di- $\boldsymbol{O}$-heptanoyl-5'- $\boldsymbol{O}$-(triphenylmethyl)cytidine (10): Yield $78.9 \%$ as crystalline solid, M.P. $98-99{ }^{\circ} \mathrm{C}\left(\mathrm{CHCl}_{3}-\mathrm{C}_{6} \mathrm{H}_{14}\right), \mathrm{R}_{f}=0.50\left(\mathrm{CHCl}_{3} / \mathrm{CH}_{3} \mathrm{OH}=24 / 1\right.$, v/v). FTIR: $v_{\max } 1715$ (-CO), $3506 \mathrm{~cm}^{-1}(-\mathrm{NH}) .{ }^{1} \mathrm{H}-\mathrm{NMR}\left(400 \mathrm{MHz}, \mathrm{CDCl}_{3}\right): \delta_{\mathrm{H}} 9.03(1 \mathrm{H}, \mathrm{s},-\mathrm{NH}), 7.30(6 \mathrm{H}, \mathrm{m}, \mathrm{Ar}-\mathrm{H}), 7.28(9 \mathrm{H}$, m, Ar-H), $7.20(1 \mathrm{H}, \mathrm{d}, \mathrm{J}=7.4 \mathrm{~Hz}, \mathrm{H}-6), 6.44\left(1 \mathrm{H}, \mathrm{d}, \mathrm{J}=3.2 \mathrm{~Hz}, \mathrm{H}-1^{\prime}\right), 5.50\left(1 \mathrm{H}, \mathrm{d}, \mathrm{J}=3.2 \mathrm{~Hz}, \mathrm{H}-2^{\prime}\right)$, $5.24\left(1 \mathrm{H}, \mathrm{dd}, \mathrm{J}=3.2\right.$ and $\left.5.4 \mathrm{~Hz}, \mathrm{H}-3^{\prime}\right), 5.06\left(1 \mathrm{H}, \mathrm{dd}, \mathrm{J}=2.2\right.$ and $\left.12.2 \mathrm{~Hz}, \mathrm{H}-5^{\prime} \mathrm{a}\right), 4.65\left(2 \mathrm{H}, \mathrm{s},-\mathrm{NH}_{2}\right)$, $4.02\left(1 \mathrm{H}, \mathrm{dd}, \mathrm{J}=2.1\right.$ and $\left.12.1 \mathrm{~Hz}, \mathrm{H}-5^{\prime} \mathrm{b}\right), 3.95\left(1 \mathrm{H}, \mathrm{dd}, \mathrm{J}=2.2\right.$ and $\left.5.3 \mathrm{~Hz}, \mathrm{H}-4^{\prime}\right), 3.80(1 \mathrm{H}, \mathrm{m}$, H-5), $2.41\left\{4 \mathrm{H}, \mathrm{m}, 2 \times \mathrm{CH}_{3}\left(\mathrm{CH}_{2}\right)_{4} \mathrm{CH}_{2} \mathrm{CO}-\right\}, 1.62\left\{4 \mathrm{H}, \mathrm{m}, 2 \times \mathrm{CH}_{3}\left(\mathrm{CH}_{2}\right)_{3} \mathrm{CH}_{2} \mathrm{CH}_{2} \mathrm{CO}-\right\}, 1.35\{12 \mathrm{H}, \mathrm{m}$, $\left.2 \times \mathrm{CH}_{3}\left(\mathrm{CH}_{2}\right)_{3} \mathrm{CH}_{2} \mathrm{CH}_{2} \mathrm{CO}-\right\}, 0.91\left\{6 \mathrm{H}, \mathrm{m}, 2 \times \mathrm{CH}_{3}\left(\mathrm{CH}_{2}\right)_{5} \mathrm{CO}-\right\}$. MS [M+1] $]^{+} 710.14$. 
Anal Calcd. for $\mathrm{C}_{42} \mathrm{H}_{53} \mathrm{O}_{7} \mathrm{~N}_{3}: \%$ C, 71.00, H, 7.10; \% found: C, 71.02, H, 7.09.

2',3'-Di-O-lauroyl-5'-O-(triphenylmethyl)cytidine (11): Yield $93.2 \%$ as a white crystalline solid, M.P. $107-109{ }^{\circ} \mathrm{C}\left(\mathrm{CHCl}_{3}-\mathrm{C}_{6} \mathrm{H}_{14}\right), \mathrm{R}_{f}=0.50\left(\mathrm{CHCl}_{3} / \mathrm{CH}_{3} \mathrm{OH}=23 / 1\right.$, v/v). FTIR: v $\max 1735$ (-CO), $3500 \mathrm{~cm}^{-1}(-\mathrm{NH}) .{ }^{1} \mathrm{H}-\mathrm{NMR}\left(400 \mathrm{MHz}, \mathrm{CDCl}_{3}\right): \delta_{\mathrm{H}} 9.00(1 \mathrm{H}, \mathrm{s},-\mathrm{NH}), 7.36(6 \mathrm{H}, \mathrm{m}, \mathrm{Ar}-\mathrm{H}), 7.31(9 \mathrm{H}, \mathrm{m}$, Ar-H), 7.28 (1H, d, J = 7.3 Hz, H-6), $6.47\left(1 \mathrm{H}, \mathrm{d}, \mathrm{J}=3.3 \mathrm{~Hz}, \mathrm{H}-1^{\prime}\right), 5.57$ (1H, d, J = 3.2 Hz, H-2'), 5.27 $\left(1 \mathrm{H}, \mathrm{dd}, \mathrm{J}=3.2\right.$ and $\left.5.4 \mathrm{~Hz}, \mathrm{H}-3^{\prime}\right), 5.11\left(1 \mathrm{H}, \mathrm{dd}, \mathrm{J}=2.1\right.$ and $\left.12.1 \mathrm{~Hz}, \mathrm{H}-5^{\prime} \mathrm{a}\right), 4.58\left(2 \mathrm{H}, \mathrm{s},-\mathrm{NH}_{2}\right), 4.11$ $\left(1 \mathrm{H}, \mathrm{dd}, \mathrm{J}=2.2\right.$ and $\left.12.2 \mathrm{~Hz}, \mathrm{H}-5^{\prime} \mathrm{b}\right), 3.99\left(1 \mathrm{H}, \mathrm{dd}, \mathrm{J}=2.1\right.$ and $\left.5.2 \mathrm{~Hz}, \mathrm{H}-4^{\prime}\right), 3.85(1 \mathrm{H}, \mathrm{d}, \mathrm{J}=7.1 \mathrm{~Hz}$, $\mathrm{H}-5), 2.37\left\{4 \mathrm{H}, \mathrm{m}, 2 \times \mathrm{CH}_{3}\left(\mathrm{CH}_{2}\right)_{9} \mathrm{CH}_{2} \mathrm{CO}-\right\}, 1.68\left\{4 \mathrm{H}, \mathrm{m}, 2 \times \mathrm{CH}_{3}\left(\mathrm{CH}_{2}\right)_{8} \mathrm{CH}_{2} \mathrm{CH}_{2} \mathrm{CO}-\right\}, 1.29\{32 \mathrm{H}, \mathrm{m}$, $\left.2 \times \mathrm{CH}_{3}\left(\mathrm{CH}_{2}\right)_{8} \mathrm{CH}_{2} \mathrm{CH}_{2} \mathrm{CO}-\right\}, 0.89\left\{6 \mathrm{H}, \mathrm{m}, 2 \times \mathrm{CH}_{3}\left(\mathrm{CH}_{2}\right)_{10} \mathrm{CO}-\right\}$. MS [M+1] $]^{+} 850.23$.

Anal Calcd. for $\mathrm{C}_{52} \mathrm{H}_{71} \mathrm{O}_{7} \mathrm{~N}_{3}$ : \% C, 73.50, H, 8.36; \% found: C, 73.49, $\mathrm{H}, 8.35$.

2',3'-Di- $\boldsymbol{O}$-myristoyl-5'- $\boldsymbol{O}$-(triphenylmethyl)cytidine (12): Yield $88.75 .3 \%$ as a crystalline solid, M.P. $96-98{ }^{\circ} \mathrm{C}\left(\mathrm{CHCl}_{3}-\mathrm{C}_{6} \mathrm{H}_{14}\right), \mathrm{R}_{f}=0.51\left(\mathrm{CHCl}_{3} / \mathrm{CH}_{3} \mathrm{OH}=24 / 1\right.$, v/v). FTIR: $v_{\max } 1726$ (-CO), $3470 \mathrm{~cm}^{-1}(-\mathrm{NH}) .{ }^{1} \mathrm{H}-\mathrm{NMR}\left(400 \mathrm{MHz}, \mathrm{CDCl}_{3}\right): \delta_{\mathrm{H}} 9.09(1 \mathrm{H}, \mathrm{s},-\mathrm{NH}), 7.38(6 \mathrm{H}, \mathrm{m}, \mathrm{Ar}-\mathrm{H}), 7.33(9 \mathrm{H}, \mathrm{m}$, Ar-H), 7.29 (1H, d, J = 7.4 Hz, H-6), $6.40\left(1 \mathrm{H}, \mathrm{d}, \mathrm{J}=3.2 \mathrm{~Hz}, \mathrm{H}-1^{\prime}\right), 5.51\left(1 \mathrm{H}, \mathrm{d}, \mathrm{J}=3.2 \mathrm{~Hz}, \mathrm{H}-2^{\prime}\right), 5.20$ $\left(1 \mathrm{H}, \mathrm{dd}, \mathrm{J}=3.1\right.$ and $\left.5.2 \mathrm{~Hz}, \mathrm{H}-3^{\prime}\right), 5.01\left(1 \mathrm{H}, \mathrm{dd}, \mathrm{J}=2.1\right.$ and $\left.12.1 \mathrm{~Hz}, \mathrm{H}-5^{\prime} \mathrm{a}\right), 4.59\left(2 \mathrm{H}, \mathrm{s},-\mathrm{NH}_{2}\right), 4.10$ $\left(1 \mathrm{H}, \mathrm{dd}, \mathrm{J}=2.1\right.$ and $\left.12.1 \mathrm{~Hz}, \mathrm{H}-5^{\prime} \mathrm{b}\right), 3.89\left(1 \mathrm{H}, \mathrm{dd}, \mathrm{J}=2.1\right.$ and $\left.5.2 \mathrm{~Hz}, \mathrm{H}-4^{\prime}\right), 3.87(1 \mathrm{H}, \mathrm{d}, \mathrm{J}=7.1 \mathrm{~Hz}$, H-5), $2.38\left\{4 \mathrm{H}, \mathrm{m}, 2 \times \mathrm{CH}_{3}\left(\mathrm{CH}_{2}\right)_{11} \mathrm{CH}_{2} \mathrm{CO}-\right\}, 1.68\left\{4 \mathrm{H}, \mathrm{m}, 2 \times \mathrm{CH}_{3}\left(\mathrm{CH}_{2}\right)_{10} \mathrm{CH}_{2} \mathrm{CH}_{2} \mathrm{CO}-\right\}, 1.29\{40 \mathrm{H}, \mathrm{m}$, $\left.2 \times \mathrm{CH}_{3}\left(\mathrm{CH}_{2}\right)_{10} \mathrm{CH}_{2} \mathrm{CH}_{2} \mathrm{CO}-\right\}, 0.95\left\{6 \mathrm{H}, \mathrm{m}, 2 \times \mathrm{CH}_{3}\left(\mathrm{CH}_{2}\right)_{12} \mathrm{CO}-\right\}$. MS [M+1] 906.04 .

Anal Calcd. for $\mathrm{C}_{56} \mathrm{H}_{79} \mathrm{O}_{7} \mathrm{~N}_{3}$ : \% C, 74.25, H, 8.73; \% found: $\mathrm{C}, 74.27, \mathrm{H}, 8.74$.

2',3'-Di- $\boldsymbol{O}$-pivaloyl-5'-O-(triphenylmethyl)cytidine (13): Yield $91.4 \%$ as crystalline solid, M.P. 96-97 ${ }^{\circ} \mathrm{C}\left(\mathrm{CHCl}_{3}-\mathrm{C}_{6} \mathrm{H}_{14}\right), \mathrm{R}_{f}=0.55\left(\mathrm{CHCl}_{3} / \mathrm{CH}_{3} \mathrm{OH}=24 / 1\right.$, v/v). FTIR: $v_{\max } 1718(-\mathrm{CO}), 3501 \mathrm{~cm}^{-1}$ (-NH). ${ }^{1} \mathrm{H}-\mathrm{NMR}\left(400 \mathrm{MHz}, \mathrm{CDCl}_{3}\right): \delta_{\mathrm{H}} 9.04(1 \mathrm{H}, \mathrm{s},-\mathrm{NH}), 7.33(6 \mathrm{H}, \mathrm{m}, \mathrm{Ar}-\mathrm{H}), 7.30(9 \mathrm{H}, \mathrm{m}, \mathrm{Ar}-\mathrm{H})$, $7.20(1 \mathrm{H}, \mathrm{d}, \mathrm{J}=7.5 \mathrm{~Hz}, \mathrm{H}-6), 6.36\left(1 \mathrm{H}, \mathrm{d}, \mathrm{J}=3.1 \mathrm{~Hz}, \mathrm{H}-1^{\prime}\right), 5.50\left(1 \mathrm{H}, \mathrm{d}, \mathrm{J}=3.1 \mathrm{~Hz}, \mathrm{H}-2^{\prime}\right), 5.10(1 \mathrm{H}, \mathrm{dd}$, $\mathrm{J}=3.2$ and $\left.5.2 \mathrm{~Hz}, \mathrm{H}-3^{\prime}\right), 5.00\left(1 \mathrm{H}, \mathrm{dd}, \mathrm{J}=2.2\right.$ and $\left.12.2 \mathrm{~Hz}, \mathrm{H}-5^{\prime} \mathrm{a}\right), 4.89\left(2 \mathrm{H}, \mathrm{s},-\mathrm{NH}_{2}\right), 4.11(1 \mathrm{H}, \mathrm{dd}$, $\mathrm{J}=2.2$ and $\left.12.1 \mathrm{~Hz}, \mathrm{H}-5^{\prime} \mathrm{b}\right), 3.92\left(1 \mathrm{H}, \mathrm{dd}, \mathrm{J}=2.2\right.$ and $\left.5.4 \mathrm{~Hz}, \mathrm{H}-4^{\prime}\right), 3.90(1 \mathrm{H}, \mathrm{d}, \mathrm{J}=7.1 \mathrm{~Hz}, \mathrm{H}-5), 1.26$ $\left\{18 \mathrm{H}, \mathrm{s}, 2 \times\left(\mathrm{CH}_{3}\right)_{3} \mathrm{CCO}-\right\}$. MS $[\mathrm{M}+1]^{+} 654.01$.

Anal Calcd. for $\mathrm{C}_{38} \mathrm{H}_{43} \mathrm{O}_{7} \mathrm{~N}_{3}$ : \% C, 69.83, $\mathrm{H}, 6.58$; \% found: $\mathrm{C}, 69.84, \mathrm{H}, 6.60$.

2',3'-Di- $\boldsymbol{O}$-(4-chlorobenzoyl)-5'-O-(triphenylmethyl) cytidine (14): Yield $78.3 \%$ as a white crystalline solid, M.P. $99-101{ }^{\circ} \mathrm{C}\left(\mathrm{CHCl}_{3}-\mathrm{C}_{6} \mathrm{H}_{14}\right), \mathrm{R}_{f}=0.52\left(\mathrm{CHCl}_{3} / \mathrm{CH}_{3} \mathrm{OH}=22 / 1\right.$, v/v). FTIR: $v_{\max }$ 1711 (-CO), $3505 \mathrm{~cm}^{-1}$ (-NH). ${ }^{1} \mathrm{H}-\mathrm{NMR}\left(400 \mathrm{MHz}, \mathrm{CDCl}_{3}\right)$ : $\delta_{\mathrm{H}} 9.01(1 \mathrm{H}, \mathrm{s},-\mathrm{NH}), 7.98(4 \mathrm{H}, \mathrm{m}, \mathrm{Ar}-\mathrm{H})$, 7.58 (4H, m, Ar-H), 7.32 (6H, m, Ar-H), 7.28 (9H, m, Ar-H), 7.21 (1H, d, J = 7.5 Hz, H-6), 6.44 (1H, d, $\left.\mathrm{J}=3.1 \mathrm{~Hz}, \mathrm{H}-\mathrm{1}^{\prime}\right), 5.58\left(1 \mathrm{H}, \mathrm{d}, \mathrm{J}=3.2 \mathrm{~Hz}, \mathrm{H}-2^{\prime}\right), 5.12\left(1 \mathrm{H}, \mathrm{dd}, \mathrm{J}=3.2\right.$ and $\left.5.2 \mathrm{~Hz}, \mathrm{H}-3^{\prime}\right), 5.02(1 \mathrm{H}, \mathrm{dd}$, $\mathrm{J}=2.2$ and $12.2 \mathrm{~Hz}, \mathrm{H}-5$ ' a), $4.60\left(2 \mathrm{H}, \mathrm{s},-\mathrm{NH}_{2}\right), 4.10\left(1 \mathrm{H}, \mathrm{dd}, \mathrm{J}=2.1\right.$ and $\left.12.1 \mathrm{~Hz}, \mathrm{H}-5^{\prime} \mathrm{b}\right), 4.00(1 \mathrm{H}, \mathrm{dd}$, $\mathrm{J}=2.2$ and $\left.5.4 \mathrm{~Hz}, \mathrm{H}-4^{\prime}\right), 3.92(1 \mathrm{H}, \mathrm{d}, \mathrm{J}=7.1 \mathrm{~Hz}, \mathrm{H}-5)$. MS $[\mathrm{M}+1]^{+} 763.22$.

Anal Calcd. for $\mathrm{C}_{42} \mathrm{H}_{33} \mathrm{O}_{7} \mathrm{~N}_{3} \mathrm{Cl}_{2}: \%$ C, 66.14, $\mathrm{H}, 4.33$; \% found: $\mathrm{C}, 66.12, \mathrm{H}, 4.32$.

$\mathbf{2}^{\prime}, \mathbf{3}^{\prime}$-Di- $\boldsymbol{O}$-cinnamoyl-5'-O -(triphenylmethyl)cytidine (15): Yield $88.5 \%$ white crystalline solid, M.P. $102-104{ }^{\circ} \mathrm{C}\left(\mathrm{CHCl}_{3}-\mathrm{C}_{6} \mathrm{H}_{14}\right), \mathrm{R}_{f}=0.50\left(\mathrm{CHCl}_{3} / \mathrm{CH}_{3} \mathrm{OH}=22 / 1, \mathrm{v} / \mathrm{v}\right)$. FTIR: $v_{\max } 1678$ (-CO), $3492 \mathrm{~cm}^{-1}(-\mathrm{NH}) .{ }^{1} \mathrm{H}-\mathrm{NMR}\left(400 \mathrm{MHz}, \mathrm{CDCl}_{3}\right): \delta_{\mathrm{H}} 9.02(1 \mathrm{H}, \mathrm{s},-\mathrm{NH}), 7.85(4 \mathrm{H}, \mathrm{m}, \mathrm{Ar}-\mathrm{H})$, $7.40(6 \mathrm{H}, \mathrm{m}, \mathrm{Ar}-\mathrm{H}), 7.60,7.57(2 \times 1 \mathrm{H}, 2 \times \mathrm{d}, \mathrm{J}=16.1 \mathrm{~Hz}, 2 \times \mathrm{PhCH}=\mathrm{CHCO}-), 7.36(6 \mathrm{H}, \mathrm{m}, \mathrm{Ar}-\mathrm{H}), 7.31$ $(9 \mathrm{H}, \mathrm{m}, \mathrm{Ar}-\mathrm{H}), 7.28(1 \mathrm{H}, \mathrm{d}, \mathrm{J}=7.4 \mathrm{~Hz}, \mathrm{H}-6), 6.45\left(1 \mathrm{H}, \mathrm{d}, \mathrm{J}=3.2 \mathrm{~Hz}, \mathrm{H}-1^{\prime}\right), 6.48,6.42(2 \times 1 \mathrm{H}, 2 \times \mathrm{d}$, $\mathrm{J}=16.1 \mathrm{~Hz}, 2 \times \mathrm{PhCH}=\mathrm{CHCO}-), 5.68\left(1 \mathrm{H}, \mathrm{d}, \mathrm{J}=3.3 \mathrm{~Hz}, \mathrm{H}-2^{\prime}\right), 5.34(1 \mathrm{H}, \mathrm{dd}, \mathrm{J}=3.3$ and $5.3 \mathrm{~Hz}$, $\left.\mathrm{H}-3^{\prime}\right), 4.97\left(1 \mathrm{H}, \mathrm{dd}, \mathrm{J}=2.2\right.$ and $\left.12.2 \mathrm{~Hz}, \mathrm{H}-5^{\prime} \mathrm{a}\right), 4.61\left(2 \mathrm{H}, \mathrm{s},-\mathrm{NH}_{2}\right), 4.31(1 \mathrm{H}, \mathrm{dd}, \mathrm{J}=2.2$ and 
$\left.12.2 \mathrm{~Hz}, \mathrm{H}-5^{\prime} \mathrm{b}\right), 4.15\left(1 \mathrm{H}, \mathrm{dd}, \mathrm{J}=2.2\right.$ and $\left.5.4 \mathrm{~Hz}, \mathrm{H}-4^{\prime}\right), 3.62(1 \mathrm{H}, \mathrm{d}, \mathrm{J}=7.1 \mathrm{~Hz}, \mathrm{H}-5) . \mathrm{MS}[\mathrm{M}+1]^{+}$ 743.30 .

Anal Calcd. for $\mathrm{C}_{46} \mathrm{H}_{36} \mathrm{O}_{7} \mathrm{~N}_{3}$ : \% C, 74.39, H, 4.85; \% found: C, 74.41, H, 4.84.

\section{X-ray powder diffraction}

X-ray powder diffraction was performed using Rigaku Dmax2200PC diffractometer (Rigaku Corporation, Tokyo, Japan) and $\mathrm{Cu} \mathrm{K} \alpha$-radiation [22]. The X-ray intensity was measured in the range of $5^{\circ} \leq 2 \theta \leq 90^{\circ}$ with a scan speed of $2^{\circ} \cdot \mathrm{min}^{-1}$. The peak position of the 002 coke peak was measured. By using Bragg's law, the interlayer d-spacing was calculated. The improved Langford method was employed to calculate the stacking disorder degree, $\mathrm{P}$.

\section{Results and discussion}

\section{Chemistry}

In this study, regioselective decanoylation (Fig. 2 and 3) and triphenylmethylation (Fig. 4 and 5) of cytidine (1) were performed using the direct method. The resulting decanoylation and triphenylmethylation products were converted into numerous analogs by employing various acylating agents.

\section{Characterization and selective decanoyl of cytidine}

Cytidine 1 was initially converted into the $5^{\prime}$ - $O$-decanoylcytidine derivative 2 through treatment with dry pyridine, and this product after the reaction with decanoyl chloride, followed by solvent removal and silica gel column chromatographic purification, produced 5 - $O$-decanoyl derivative (2) with $83.8 \%$ yield as needles and m.p. of $85-87^{\circ} \mathrm{C}$ ). The FTIR spectrum of compound 2 showed the following absorption bands: $1731,1714 \mathrm{~cm}^{-1}$ (due to $-\mathrm{CO}$ ), $3420 \mathrm{~cm}^{-1}$ (due to $-\mathrm{OH}$ ), and $3550 \mathrm{~cm}^{-1}$ (due to $-\mathrm{NH}$ ) stretching. In its ${ }^{1} \mathrm{H}-\mathrm{NMR}$ spectrum, two two-proton multiplets observed at $\delta 2.38$ $\left\{\mathrm{CH}_{3}\left(\mathrm{CH}_{2}\right)_{7} \mathrm{CH}_{2} \mathrm{CO}-\right\}$ and $\delta 1.59\left\{\mathrm{CH}_{3}\left(\mathrm{CH}_{2}\right)_{6} \mathrm{CH}_{2} \mathrm{CH}_{2} \mathrm{CO}-\right\}$, a twelve-proton multiplet appearing at $\delta 1.32\left\{\mathrm{CH}_{3}\left(\mathrm{CH}_{2}\right)_{6}\left(\mathrm{CH}_{2}\right)_{2} \mathrm{CO}-\right\}$, and a three-proton multiplet seen at $\delta 0.85\left\{\mathrm{CH}_{3}\left(\mathrm{CH}_{2}\right)_{8} \mathrm{CO}-\right\}$ were caused by the presence of one decanoyl group in the molecule. The downfield shift of $\mathrm{C}-5^{\prime}$ proton to $\delta 6.01$ (as dd, $\mathrm{J}=2.4$ and $12.3 \mathrm{~Hz}, \mathrm{H}-5^{\prime} \mathrm{a}$ ) and to $\delta 5.56$ (as dd, $\mathrm{J}=2.4$ and $12.3 \mathrm{~Hz}, \mathrm{H}-5^{\prime}$ b) from their general values [23] in the precursor compound (1) and the resonances of other protons in their anticipated positions indicated the presence of the decanoyl group at position $5^{\prime}$. The formation of 5 - $O$-decanoylcytidine (2) might be caused by the high reactivity of the sterically less hindered

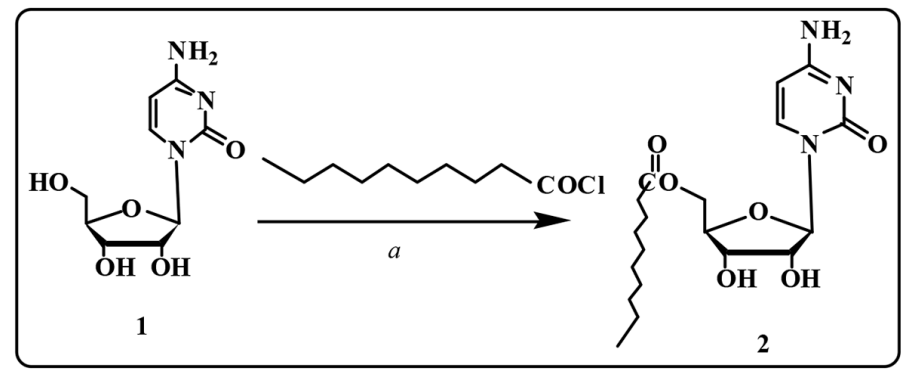

Fig. 2. Reagents and conditions: (a) dry $\mathrm{C}_{6} \mathrm{H}_{5} \mathrm{~N},-5^{\circ} \mathrm{C}, 6$ to $7 \mathrm{~h} ; \mathbf{2}=$ decanoyl derivative 


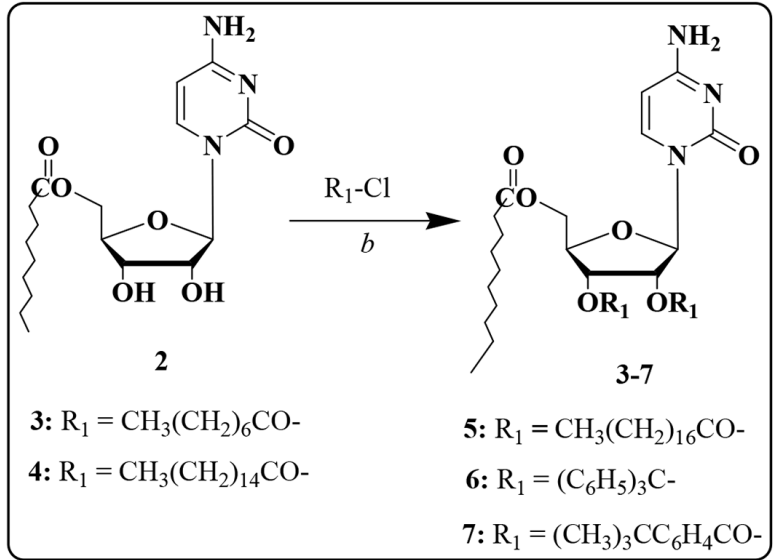

Fig. 3. (b) dry pyridine, $0{ }^{\circ} \mathrm{C}$ to room temperature, DMAP, stir for $6-8 \mathrm{~h}, \mathrm{R}_{1}=$ different acyl halides (3-7)

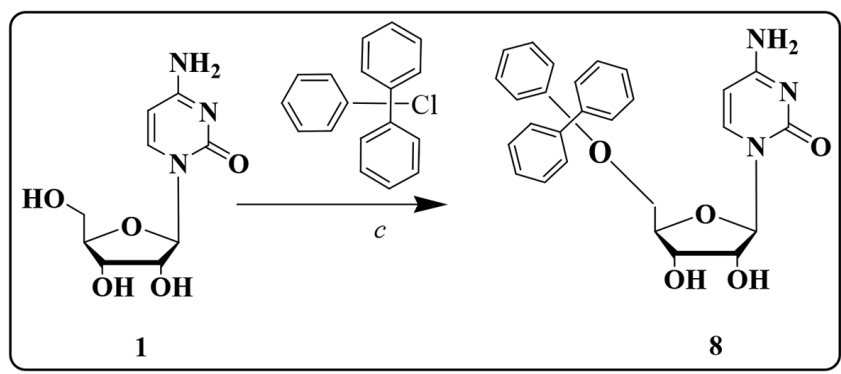

Fig. 4. Reagents and conditions: $(c)$ dry $\mathrm{C}_{6} \mathrm{H}_{5} \mathrm{~N}, 0-5^{\circ} \mathrm{C}, 6 \mathrm{~h} ; \mathbf{8}=$ triphenylmethyl derivative

primary hydroxyl group of the ribose moiety of cytidine (1). Mass spectrometry provided a molecular ion peak at $m / z[\mathrm{M}+1]^{+} 398.10$, which corresponded to the aforementioned molecular formula. From the complete analysis of FTIR, ${ }^{1} \mathrm{H}-\mathrm{NMR}$, and elemental data, the structure of this compound was assigned as 5'-O-decanoylcytidine (2).

Furthermore, the structure of compound (2) was confirmed through the preparation of its octanoyl derivatives (3) $(77.6 \%)$ as the crystalline solid with the m.p. of $89-91{ }^{\circ} \mathrm{C}$. In its ${ }^{1} \mathrm{H}-\mathrm{NMR}$ spectrum, two four-proton multiplets appearing at $\delta 2.37\left\{2 \times \mathrm{CH}_{3}\left(\mathrm{CH}_{2}\right)_{5} \mathrm{CH}_{2} \mathrm{CO}-\right\}$ and $1.64\{2 \times$ $\left.\mathrm{CH}_{3}\left(\mathrm{CH}_{2}\right)_{4} \mathrm{CH}_{2} \mathrm{CH}_{2} \mathrm{CO}-\right\}$, sixteen-proton multiplet observed at $\delta 1.29\left\{2 \times \mathrm{CH}_{3}\left(\mathrm{CH}_{2}\right)_{4}\left(\mathrm{CH}_{2}\right)_{2} \mathrm{CO}-\right\}$, and six-proton multiplet obtained at $\delta 0.89\left\{2 \times \mathrm{CH}_{3}\left(\mathrm{CH}_{2}\right)_{6} \mathrm{CO}-\right\}$ were caused by the presence of two octanoyl groups in the molecule. The downfield shift of $\mathrm{H}^{-} 2^{\prime}$ and $\mathrm{H}-3^{\prime}$ protons to $\delta 5.45$ and $\delta 4.82$ from their precursor values (2) [24] indicated the attachment of two octanoyl groups at positions $2^{\prime}$ and 3'. The structure of octanoyl derivatives (3) was confirmed as $5^{\prime}$ - $O$-decanoyl-2',3'-di- $O$-octanoylcytidine (3)through the complete analysis of their FTIR, ${ }^{1} \mathrm{H}-\mathrm{NMR}$, and elemental data.

Through the palmitoylation of compound (2) by using palmitoyl chloride as acylating agent in $\mathrm{C}_{5} \mathrm{H}_{5} \mathrm{~N}$ at room temperature, we isolated compound 4 in good yield. The following resonance peaks ascertained the presence of two palmitoyl groups in the molecule: $\delta 2.36\left\{4 \mathrm{H}, \mathrm{m}, 2 \times \mathrm{CH}_{3}\left(\mathrm{CH}_{2}\right)_{13} \mathrm{CH}_{2} \mathrm{CO}-\right\}$, $\delta 1.27\left\{52 \mathrm{H}, \mathrm{m}, 2 \times \mathrm{CH}_{3}\left(\mathrm{CH}_{2}\right)_{13} \mathrm{CH}_{2} \mathrm{CO}-\right\}$, and $\delta 0.91\left\{6 \mathrm{H}, \mathrm{m}, 2 \times \mathrm{CH}_{3}\left(\mathrm{CH}_{2}\right)_{14} \mathrm{CO}-\right\}$. The introduction of palmitoyl groups at position $2^{\prime}$ and $3^{\prime}$ was indicated by appearance of $\mathrm{H}-2^{\prime}$ and $\mathrm{H}-3^{\prime}$ resonance peaks 
at $\delta 5.48$ (as m) and $\delta 4.86$ (as dd, $\mathrm{J}=3.5$ and $5.6 \mathrm{~Hz}$ ), which were deshielded considerably from their precursor diol (2) peaks. The decanoyl derivative 2 was further transformed easily into the $2^{\prime}, 3^{\prime}$-di- $O$ stearoyate 5, 2',3'-di-O-(triphenylmethyloate) 6, and 2',3'-(4-tert-butylbenzoate) 7.

\section{Characterization and selective triphenylmethylation of cytidine}

Cytidine (1) was then transformed into the $5^{\prime}-O$-(triphenylmethyl)cytidine derivative 8 through a treatment with a unimolecular amount of triphenylmethyl chloride in anhydrous pyridine at $-5{ }^{\circ} \mathrm{C}$. The conventional work-up procedure, followed by solvent removal and silica gel column chromatographic purification, produced high yields of the triphenylmethyl derivative $(\mathbf{8})$ as the crystalline solid. In its ${ }^{1} \mathrm{H}-\mathrm{NMR}$ spectrum, two characteristic six-proton multiplets appearing at $\delta 7.35(\mathrm{Ar}-\mathrm{H})$ and nineproton multiplets observed at $\delta 7.30(\mathrm{Ar}-\mathrm{H})$ were caused by three phenyl protons of the triphenylmethyl group in the molecule. The downfield shift of C-5/ proton to $\delta 5.30$ (as dd, J $=2.2$ and $12.2 \mathrm{~Hz}, \mathrm{H}-5^{\prime} \mathrm{a}$ ) and to 5.26 (as dd, $J=2.2$ and $12.2 \mathrm{~Hz}, \mathrm{H}-5^{\prime} \mathrm{b}$ ) from their usual values ( $\sim 4.00 \mathrm{ppm}$ ) in the precursor compound (1) and the resonances of other protons at their anticipated positions showed the presence of the triphenylmethyl group at position 5. This finding is in accordance with the mechanism proposed by Kawsar et al. [25] based on similar nucleoside derivatives.

The preparation and identification of hexanoyl derivative 9 further supported the structure of compound 8. The ${ }^{1} \mathrm{H}-\mathrm{NMR}$ spectra exhibited two four-proton multiplets at $\delta 2.31$ $\left\{2 \times \mathrm{CH}_{3}\left(\mathrm{CH}_{2}\right)_{3} \mathrm{CH}_{2} \mathrm{CO}-\right\}$ and at $\delta 1.62\left\{2 \times \mathrm{CH}_{3}\left(\mathrm{CH}_{2}\right)_{2} \mathrm{CH}_{2} \mathrm{CH}_{2} \mathrm{CO}-\right\}$, eight-proton multiplet at $\delta 1.26$ $\left\{2 \times \mathrm{CH}_{3}\left(\mathrm{CH}_{2}\right)_{2} \mathrm{CH}_{2} \mathrm{CH}_{2} \mathrm{CO}-\right\}$, and six-proton multiplet at $\delta 0.88\left\{2 \times \mathrm{CH}_{3}\left(\mathrm{CH}_{2}\right)_{4} \mathrm{CO}-\right\}$, which showed the attachment of two hexanoyl groups indicating the formation of $2^{\prime}, 3^{\prime}$-di- $O$-hexanoate 9.

Compound 8 was then converted into heptanoyl derivative 10 by using similar procedures, and a high yield of heptanoate $\mathbf{1 0}$ was isolated as needles, (m.p. $98-99^{\circ} \mathrm{C}$ ). From the complete analysis of the FTIR, ${ }^{1} \mathrm{H}-\mathrm{NMR}$, and elemental data, the structure of this compound was confirmed as $2^{\prime}, 3^{\prime}$-di- $O$ heptanoyl -5'-O-(triphenylmethyl)cytidine (10). Similarly, compound 8 was converted into numerous acylated derivatives (11-15) to obtain newer compounds for antimicrobial evaluation studies. The structures of these derivatives were ascertained through the complete interpretation of their FTIR and ${ }^{1} \mathrm{H}-\mathrm{NMR}$ spectra.

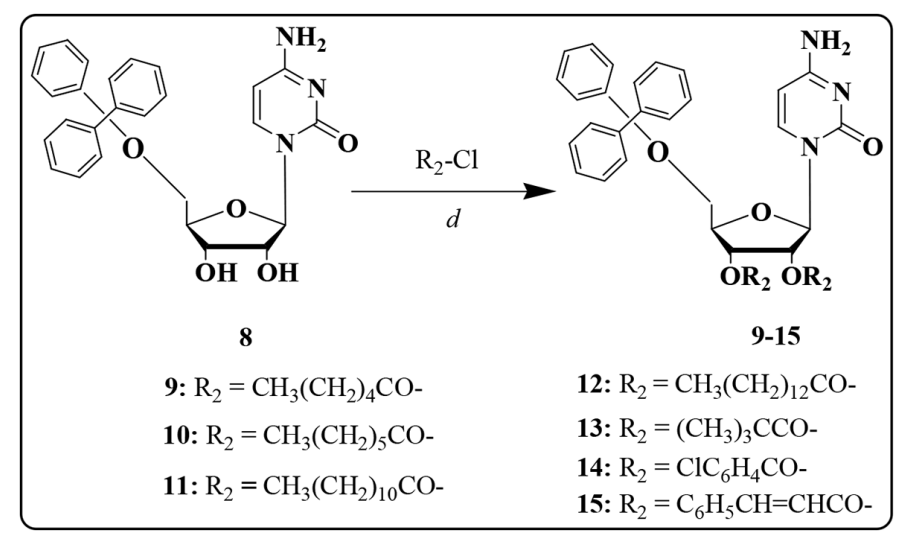

Fig. 5. Reagents and conditions: $(d)$ anhydr. pyridine, $0{ }^{\circ} \mathrm{C}$ to room temperature, DMAP, stir for 6 to $7 \mathrm{~h}$, $\mathrm{R}_{2}=$ several acyl halides $(\mathbf{9}-\mathbf{1 5})$ 
Thus, cytidine (1) acylation by applying the direct method was unique, and the reaction provided a single mono-substituted product in reasonably high yields. These newly synthesized products may be used as important precursors to modify cytidine molecules at different positions. All the prepared products were employed as test compounds for evaluating their antimicrobial and anticancer activities and for computational investigations.

\section{XRD measurements}

$\mathrm{XRD}$ is mainly performed for quantitatively identifying crystalline compounds, whereas single crystal XRD is conducted for structure determination. If $\mathrm{h}, \mathrm{k}$, and 1 represent the miller indices, the rules of the determination of crystal lattice type are as follows (Table 1).

The XRD patterns of the pure compounds synthesized under the optimized conditions were obtained in the $2 \theta$ range of $\left(0^{\circ}-50^{\circ}\right)$. The peaks observed at $2 \theta$ of 19.653 and 21.506 (h,k,l:112 \& 220), 6.010 and 21.472 (h,k,l:320 \& 100), 8.386 and 20.212 (h,k,l:100 \& 100), and 7.427 and 12.360 (h,k,l:100 \& 111) corresponded to compounds $4, \mathbf{5}, \mathbf{1 3}$, and $\mathbf{1 5}$, respectively. These peaks indicated the formation of typical phases of compounds $4, \mathbf{5}, \mathbf{1 3}$, and $\mathbf{1 5}$. According to the phase analysis, the compounds synthesized using this method have high purity, and no impurities were detected in the XRD pattern. Moreover, compounds 4, 5, 13, and 15 show many lines with high intensity in their XRD patterns, which indicated that all the compounds are highly crystalline (Fig. 6). By applying the rules (Table 1) of the determination of the lattice type, we assigned the lattice structures to the synthesized

Table 1. Rules of the determination of crystal lattice type

\begin{tabular}{|c|c|}
\hline Lattice type & Rules for reflection to be observed \\
\hline Primitive, $\mathrm{P}$ & None \\
\hline Body centered, $\mathrm{h}$ & $\mathrm{hkl} ; \mathrm{h}+\mathrm{k}+\mathrm{l}=2 \mathrm{n}$ \\
\hline Face centered, $\mathrm{F}$ & $\mathrm{hkl} ; \mathrm{h}, \mathrm{k}, \mathrm{l}$ either all odd or all even \\
\hline Side centered, $\mathrm{C}$ & $\mathrm{hkl} ; \mathrm{h}+\mathrm{k}=2 \mathrm{n}$ \\
\hline Rhombohedral & $\mathrm{hkl} ; \mathrm{h}+\mathrm{k}+\mathrm{l}=3 \mathrm{n}$ or $\mathrm{h}-\mathrm{k}+\mathrm{l}=3 \mathrm{n}$ \\
\hline
\end{tabular}

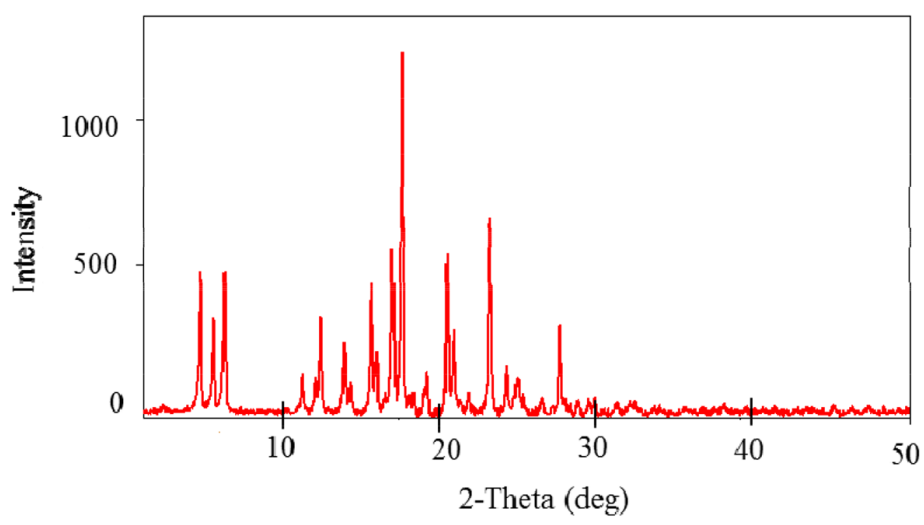

Fig. 6. XRD pattern of 2',3'-di- $O$-pivaloyl-5'-O-(triphenylmethyl)cytidine (13) 
compounds. Compound 4 satisfied the rule, $\mathrm{h}+\mathrm{k}+\mathrm{l}=2 \mathrm{n}$ and was assigned the body centered lattice, while compounds $\mathbf{5}, \mathbf{1 3}$, and 15 were assigned primitive, for which no rule was provided.

\section{Conclusion}

In conclusion, an efficient method was proposed for synthesizing cytidine analogs. Moreover, acylation reactions are highly promising because considerably high yields of a single mono-substituted product were isolated through all the reactions. XRD pattern showed that the compounds 4, 5, 13 and 15 exhibited many lines with high intensity and suggested these compounds are well crystalline.

\section{Author Contributions}

S.M.A.K. designed and planned the experiments; K.M.R., and A.H. performed the synthetic experiment and determined XRD. S.M.A.K. interpreted the data and wrote the paper. All authors have read and approved the final version of the manuscript.

\section{Acknowledgment}

The authors are grateful to the Ministry of Science and Technology (MOST), the Government of the People's Republic of Bangladesh for providing financial support (Ref:: 39.00.0000. 09.06.024.19/ Phy's-544-560, 2019-2020) to conduct this research.

\section{Declaration of interest}

The authors declare no conflict of interest.

\section{References}

1. Katherine L., Seley R., Mary K.Y. The evolution of nucleoside analogue antivirals: A review for chemists and non-chemists. Part 1: Early structural modifications to the nucleoside scaffold. Antiviral Research 2018. Vol. 154, P. 66-86.

2. Vijaya L.D., Sambasivarao D., James D.Y., Stephen A.B., John M., Michael B.S., Carol E.C. Nucleoside anticancer drugs: the role of nucleoside transporters in resistance to cancer chemotherapy. Oncogene 2003. Vol. 22, P. 7524-7536.

3. Ichikawa E., Kato K. Sugar-modified nucleosides in past 10 years, a review. Current Medicinal Chemistry 2001, Vol. 8(4), P. 385-423.

4. Emmanuel T., Marc G.G., Liang T.J. The application and mechanism of action of ribavirin in therapy of hepatitis C. Antiviral Chemistry Chemotherapy 2012. Vol. 23(1), 1-12.

5. Sidwell R.W., Huffman J.H., Khare G.P., Allen L.B., Witkowski J.T., Robins R.K.

Broad-spectrum antiviral activity of Virazole: 1-beta-D-ribofuranosyl-1,2,4-triazole-3carboxamide. Science 1972. Vol. 177(4050), P. 705-716.

6. Judith K.C. 5-Azacytidine and 5-aza-2'-deoxycytidine as inhibitors of DNA methylation: mechanistic studies and their implications for cancer therapy. Oncogene 2002. Vol. 21, P. 5483-5495.

7. Szebeni J., Wahl S.M., Betageri G.V., Wahl L.M., Gartner S., Popovic M., Parker R.J., Black C.D., Weinstein J.N. Inhibition of HIV-1 in monocyte/macrophage cultures by 2',3'-dideoxycytidine-5'-triphosphate, free and in liposomes. AIDS Research and Human Retroviruses 1990. Vol. 6(5), P. 691-702. 
8. Peter L.A., Joseph E.R. Zidovudine and Lamivudine for HIV Infection. Clinical Medical Reviews and Therapeutics 2010. Vol. 2, P. a2004-1-19.

9. Gnann J.W.J., Barton N.H., Whitley R.J. Acyclovir: mechanism of action, pharmacokinetics, safety and clinical applications. Pharmacotherapy 1983. Vol. 3(5), P. 275-283.

10. Wurtman R.J., Regan M., Ulus I., Yu L. Effect of oral CDP-choline on plasma Chlorine and Uridine levels in humans. Biochemical Pharmacology 2000. Vol. 60(7), P. 989-992.

11. Lisa A.T., Richard J.W. Dietary cytidine (5')-diphosphocholine supplementation protects against development of memory deficits in aging rats. Progress Neuro-Psychopharmacology \& Biology Psychiatry 2003. Vol. 27(4), P. 711-717.

12. Hirojuki H., Horoshi A., Hiromichi T., Tadashi M. Introduction of an alkyl group into the sugar portion of uracilnucleosides by the use of Gilman reagents. Chemical \& Pharmaceutical Bulletin 1990. Vol. 38, P. 355-360.

13. Williams J.M., Richardson A.C. Selective Acylation of pyranosides-I. benzoylation of methyl $\alpha$-D-gly- copyranosides of mannose, glucose and galactose. Tetrahedron 1967. Vol. 23, P. 1369-1378.

14. Nanda D.S., Peter D., Lisa M.S., Krishna U. A simple method for N-acylation of adenosine and cytidine nucleosides using carboxylic acids activated In-Situ with carbonyldiimidazole. Tetrahedron Letters 1995. Vol. 36(51), P. 9277-9280.

15. Bhat V., Ugarkar B.G., Sayeed V.A., Grimm K., Kosora N., Domenico P.A., Stocke E. A Simple and convenient method for the selective $\mathrm{N}$-acylations of cytosine nucleosides. Nucleosides and Nucleotides 1989. Vol. 8(2), P. 179-183.

16. Kabir A.K.M.S., Dutta P., Anwar M.N. Synthesis of some new derivatives of D-mannose. Chittagong University Journal of Science 2005. Vol. 29, P. 01-08.

17. Juraj K., Michal T., Radek P., Jan H., Petr D., Marián H., Michal H. Sugar modified pyrimido[4,5-b]indole nucleosides: synthesis and antiviral activity. Medicinal Chemistry Communications 2017. Vol. 8, P. 1856-1862.

18. Mohamed A.M., Al-Qalawi H.R., El-Sayed W.A., Arafa W.A., Alhumaimess M.S., Hassan A.K. Anticancer activity of newly synthesized triazolopyridine derivatives and their nucleoside analogs. Acta Poloniae Pharmaceutica 2015. Vol. 72, P. 307-318.

19. Devi S.R., Jesmin S., Rahman M., Manchur M.A., Fujii Y., Kanaly R.A., Ozeki Y., Kawsar S.M.A. Microbial efficacy and two step synthesis of uridine derivatives with spectral characterization. ACTA Pharmaceutica Sciencia 2019. 57, P. 47-68.

20. Kawsar S.M.A., Islam M., Jesmin S., Manchur M.A., Hasan I., Rajia S. Evaluation of the antimicrobial activity and cytotoxic effect of some uridine derivatives. International Journal of Bioscience 2018. Vol. 12, P. 211-219.

21. Shagir A.C., Bhuiyan M.M.R, Ozeki Y., Kawsar S.M.A. Simple and rapid synthesis of some nucleoside derivatives: structural and spectral characterization. Current Chemistry Letters 2016. Vol. 5, P. 83-92.

22. Said S.A.J., Anwar U.H., Abdul R.I. Mohammed, S.S. Use of X-ray powder diffraction for quantitative analysis of carbonate rock reservoir samples. Powder Technology 2007. Vol. 175, P. 115-121.

23. Arifuzzaman M., Islam M.M., Rahman M.M., Mohammad A.R., Kawsar S.M.A. An efficient approach to the synthesis of thymidine derivatives containing various acyl groups: characterization and antibacterial activities. ACTA Pharmaceutical Science 2018. Vol. 56, P. 7-22. 
24. Kawsar S.M.A., Faruk M.O., Mostafa G., Rahman M.S. Synthesis and Spectroscopic Characterization of Some Novel Acylated Carbohydrate Derivatives and Evaluation of Their Antimicrobial Activities. Chemistry \& Biology Interface 2014. Vol. 4, P. 37-47.

25. Kawsar S.M.A., Hamida A.A., Sheikh A.U., Hossain M.K., Shagir A.C., Sanaullah A.F.M., Manchur M.A., Imtiaj H., Ogawa Y., Fujii Y., Koide Y., Ozeki Y. Chemically modified uridine molecules incorporating acyl residues to enhance antibacterial and cytotoxic activities. International Journal of Organic Chemistry 2015. Vol. 5, P. 232-245. 